\title{
Prophylactic Nasogastric Decompression after Emergency Laparotomy
}

Ranjan Sapkota, ${ }^{1}$ Ramesh Singh Bhandari'

'Department of Surgery, Tribhuvan University Teaching Hospital, Kathmandu, Nepal.

Introduction: Post-operative nasogastric intubation after emergency laparotomy is a common practice in many centers, with the intent of hastening the return of bowel function, relieving gastrointestinal discomfort, reducing various post-operative complications and reducing hospital stay. However, bowel rest and gastric decompression have been re-examined in the light of more recent data. Many studies and meta-analyses over the last 50 years have challenged the routine use of nasogastric tubes after laparotomy. The objective of this study is to evaluate the need for routine nasogastric decompression after emergency laparotomy.

Methods: A prospective, randomized controlled trial was conducted for 12 months (May 1, 2007 to Apr 30, 2008) in the Department of Surgery, Tribhuvan University Teaching Hospital, after ethical approval. Patients were enrolled as per criteria (Box 1), and subsequently allocated by simple randomization into two groups: Group 1 and Group 2. Patients undergoing emergency laparotomy for perforation peritonitis, intestinal obstruction and abdominal trauma were randomized to two groups - with or without nasogastric tube after surgery. Gastric upset, return of bowel function and postoperative complications were compared.

Results: Total of 115 patients met the inclusion criteria. There was no statistically significant difference in the occurrence of gastric upset (P: 0.38), wound complications (P: 0.30), respiratory complications (P: 0.30) and anastomotic leak (P: 0.64) between two groups. Bowel function returned in comparable times in both groups (correlation coefficient: 0.14; P: 0.54). Nasogastric tube had to be reinserted in three patients in the group with nasogastric decompression postoperatively, and four in the group without (P: 0.43). Thus, routine nasogastric decompression neither prevented the development of gastrointestinal discomfort nor precluded the need for tube replacement once it was discontinued. For every patient who required post-operative nasogastric decompression, at least 14 patients were spared one. Mean hospital stay was significantly more in the decompressed group (7.52 days; correlation coefficient: $0.22 ; \mathrm{P}<0.05)$.

Conclusion: This study has shown that the prophylactic nasogastric decompression following emergency laparotomy is ineffective in achieving any of the intended goals.

Keywords: complications; decompression; emergency laparotomy; flatus; nasogastric tube; prophylactic.

Correspondence: Dr. Ranjan Sapkota, Department of Surgery, Tribhuvan University Teaching Hospital, Kathmandu, Nepal. Email: ranjansapkota@gmail.com. Phone: 9851135495. 


\section{INTRODUCTION}

For more than 300 years, Nasogastric Intubation (NGI) has been carried out for feeding, therapeutic, diagnostic or prophylactic purposes. ${ }^{1}$ Historically, all patients having abdominal operations routinely awoke with an NG tube which remained until the resolution of the postoperative ileus. ${ }^{2}$ Over several decades, this practice evolved, with the intent to hasten bowel function, to ease respiration and to diminish the risk of aspiration of gastric contents, thereby decreasing the risk of pulmonary complications. It was also meant to increase patient comfort by lessening abdominal distension, to prevent anastomotic leakage and wound dehiscence, and to shorten hospital stay. ${ }^{1}$

However, surgeons soon started questioning the utility of its routine use, and patients started asking their physicians why they need NGI. ${ }^{3}$ After 1963, Gerber and others strongly opposed routine NG decompression after abdominal operation, and rather considered it a cause of various complications. ${ }^{4}$

NG tube removes swallowed air, saliva and gastric content; but has minimal effect on the 4-5 $\mathrm{L}$ of intestinal secretion, pancreatic secretion and bile. ${ }^{5}$ Further, alimentary secretions decrease after abdominal operations. ${ }^{4,6}$ The fasting state and NG decompression can in fact contribute to post-operative ileus. ${ }^{7}$ Moreover, few patients would argue that NG tube is one of the most unpleasant aspects of their postoperative course.

NG decompression has been reexamined in light of more recent data by many investigators; seeking to reevaluate its use. ${ }^{2}$ But, despite growing evidence to the contrary, many practitioners continue to apply them routinely in the care of their patients. ${ }^{8,9}$

\section{METHODS}

A prospective, randomized, controlled study was conducted for 12 months (May 1, 2007 to Apr 30, 2008) in the Department of Surgery, Tribhuvan University Teaching Hospital, after ethical approval. Patients were enrolled as per criteria (Box 1),

Box 1. Inclusion and exclusion criteria

Inclusion criteria
All patients undergoing emergency laparotomy for
perforation peritonitis, intestinal obstruction and
abdominal trauma.
Exclusion criteria
History of abdominal/pelvic irradiation, extensive
fibrotic adhesions, operating time more than 5 hours,
requiring ventilator support postoperatively, and
denial of consent for randomization and participation.

and subsequently allocated by simple randomization into two groups: Group 1 and Group 2.
FG 16 Polyvinyl chloride NG tubes were inserted before or during operation as needed, and drained free. Gastric contents were sucked out at the completion of surgery. Group 1 patients had the NG tube in situ until the return of bowel function. Group 2 had the tube removed at the completion of surgery. NG tube was reinserted in both groups whenever deemed necessary by the treating surgeon, based on significant nausea, vomiting, abdominal distension and discomfort.

The primary endpoints (return of bowel function and gastric upset) and secondary endpoints (wound complications, respiratory complications, anastomotic leakage and length of hospital stay) were defined at the outset. (Box 2)

\section{Box 2. Study Definitions}

Return of bowel function: time for passage of first
flatus after operation
Gastric upset: nausea, vomiting or abdominal
discomfort
Wound complications: purulent discharge/dehiscence
with or without fever
Respiratory complications: atelectasis/pneumonia
with or without fever
Anastomotic leakage: drainage of Gl content at the
wound/drain
Length of hospital stay: number of postoperative
days in hospital, excluding the day of surgery
Discharge criteria: normal diet, full ambulation, oral
analgesics

Presence of gastric upset was noted by direct observation, patient's complaints and observation of the nursing staff. Abdomen was examined for the presence of bowel sounds twice a day. At the same time, patients were enquired about the approximate time range of their first flatus. Wound status was followed till suture removal in the ward or in the surgical out-patient clinic on follow up. Respiratory system examination was carried out every day before the morning rounds and as required, and chest X-ray obtained as necessary.

Data was analyzed with Statistical Package for Social Sciences (SPSS) version 14.0. Chi-square (Pearson's and Fischer's exact) tests were used for discrete variables. Numerical data and means were analyzed with one-way Analysis of variance (ANOVA). Percentages were presented with $95 \%$ confidence interval $(\mathrm{Cl})$. $\mathrm{P}$ value $<0.05$ was considered significant.

\section{RESULTS}

Out of 138 patients who underwent emergency laparotomy during the study period, only 115 entered final analysis (Figure 1). 


\section{Figure 1. Study flowchart}

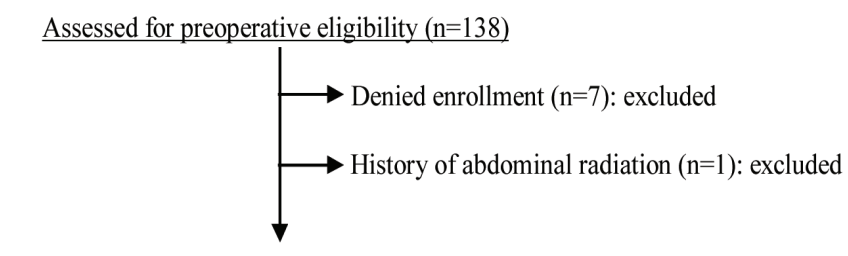

Informed consent taken for randomization $(\mathrm{n}=130)$
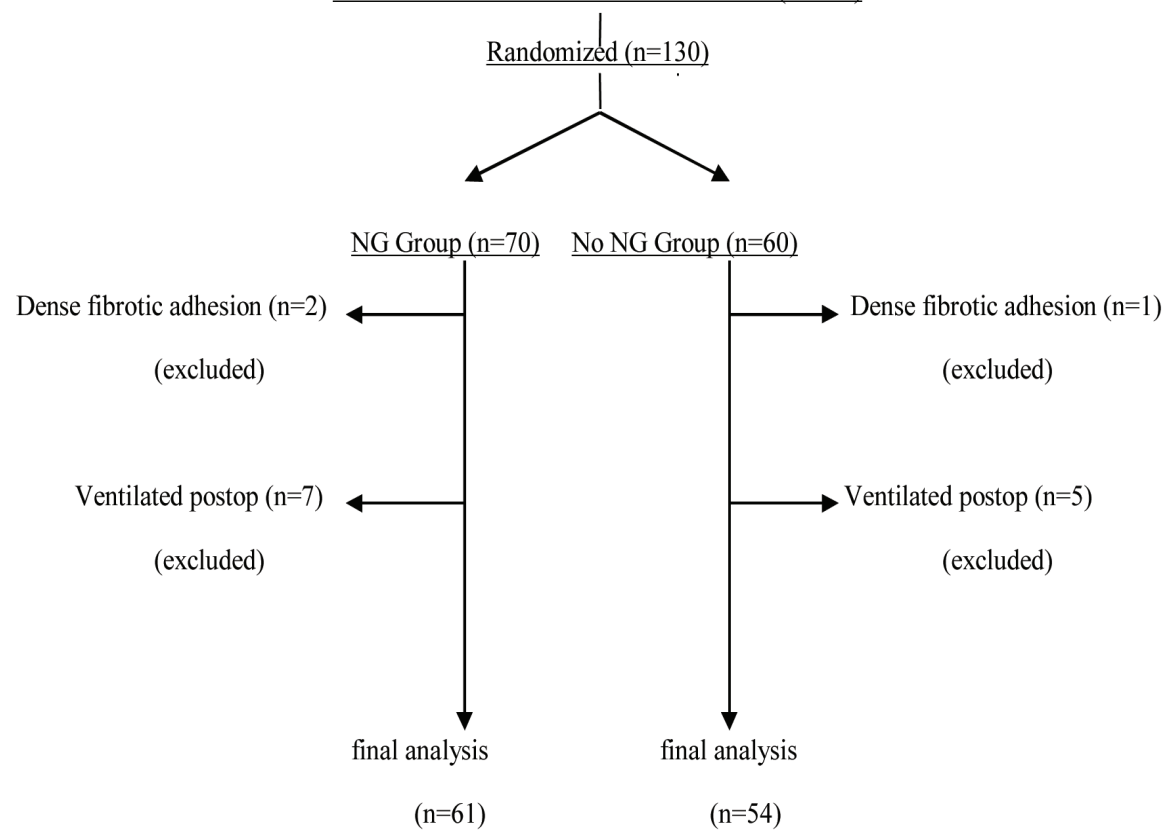

Figure 1. Study flowchart.

Both groups were comparable by baseline characteristics, including the diagnosis and surgical procedures (Table 1). Males (83) outnumbered females (32) with the mean age of $41.3 \mathrm{yr}$ (range: 16-70; SD: 14.6). Perforation
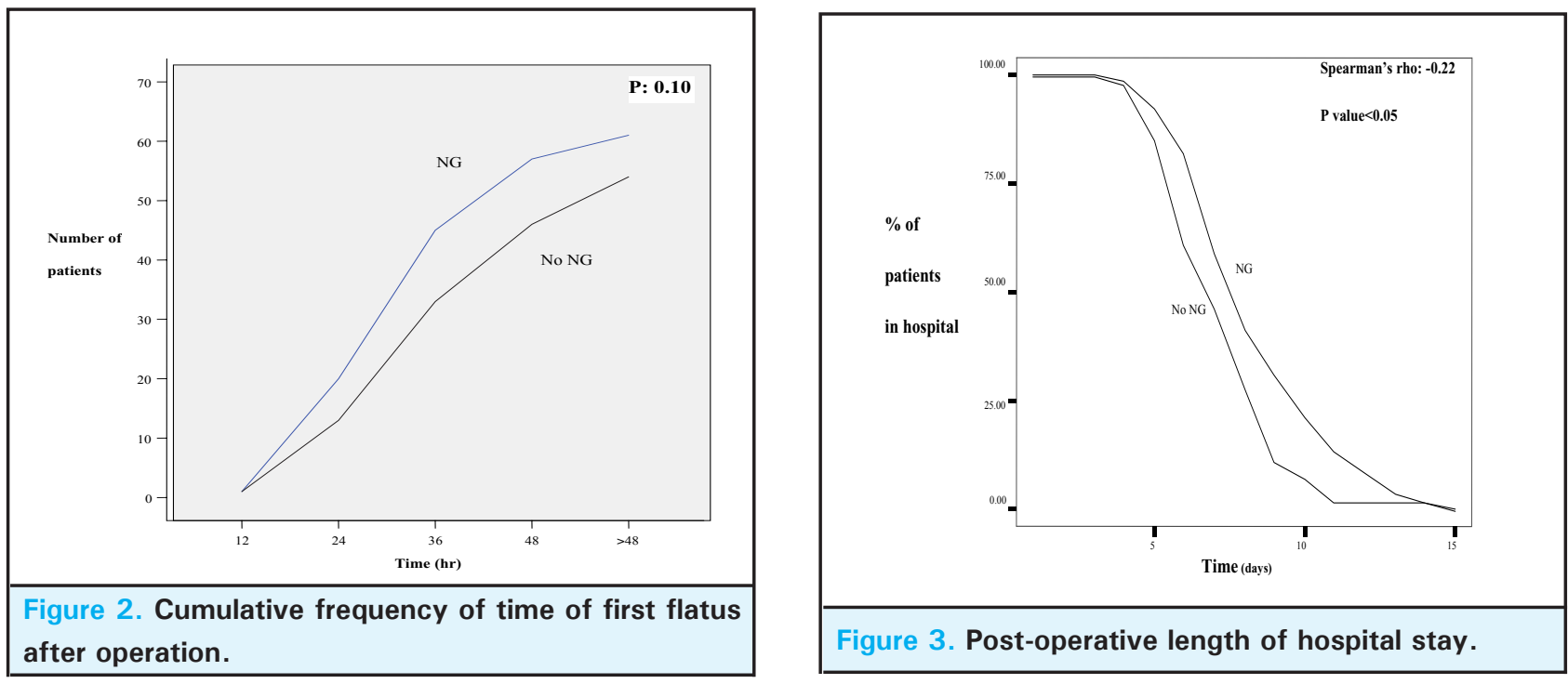


\begin{tabular}{|c|c|c|c|c|}
\hline \multicolumn{2}{|l|}{ Particulars } & Group 1 & Group 2 & p value \\
\hline \multicolumn{2}{|l|}{ Age $(y r)$, Mean $\pm S D$} & $41.08 \pm 14.6$ & $41.69 \pm 14.9$ & 0.82 \\
\hline \multirow{5}{*}{$\begin{array}{l}\text { Proportion of specific } \\
\text { patient population }\end{array}$} & Perforation peritonitis & 49.2 & 48.1 & \multirow{5}{*}{0.98} \\
\hline & Intestinal obstruction & 10.2 & 11.8 & \\
\hline & Trauma with bowel injury & 11.5 & 11.1 & \\
\hline & Trauma without bowel injury & 8.2 & 7.4 & \\
\hline & Miscellaneous & 4.9 & 5.6 & \\
\hline \multicolumn{2}{|l|}{ Sex Ratio (M/F) } & 2.5 & 2.6 & 0.57 \\
\hline \multicolumn{2}{|c|}{ Mean duration of operation, min (SD) } & $106.8(24.5)$ & $98.6(23)$ & 0.06 \\
\hline \multicolumn{2}{|c|}{ Proportion of patients with abdominal drains } & 90.2 & 92.6 & 0.64 \\
\hline \multicolumn{2}{|c|}{$\begin{array}{l}\text { Proportion of patients with bowel resection/repair/ } \\
\text { anastomosis }\end{array}$} & 72.1 & 72.2 & 0.57 \\
\hline
\end{tabular}

\begin{tabular}{|c|c|c|}
\hline Surgery & n (group 1) & n (group 2) \\
\hline $\begin{array}{l}\text { Repair of duodenal } \\
\text { ulcer perforation }\end{array}$ & 14 & 12 \\
\hline $\begin{array}{l}\text { Wedge resection and } \\
\text { repair }\end{array}$ & 5 & 6 \\
\hline $\begin{array}{l}\text { Segmental resection } \\
\text { and anastomosis }\end{array}$ & 14 & 12 \\
\hline $\begin{array}{l}\text { Lavage and } \\
\text { appendectomy }\end{array}$ & 11 & 9 \\
\hline
\end{tabular}

peritonitis was the commonest $(48.1 \%)$ diagnosis. Most operations (72.2\%) involved some form of bowel resection, repair or anastomosis (Table 2 ).

Sixty percent of the patients had at least one of the postoperative complications studied. Gastric upset was the most common one in both groups, occurring in $36 \%$ of all patients. NGI slightly increased the risks of wound complications, though not statistically significant. None of the other post-operative complications differed significantly in the two groups (Table 3).

Bowel sounds appeared in all patients by 48 hours of operation, and most patients $(88.1 \%)$ had their first bowel sounds before 24 hours. Nearly $90 \%$ patients passed their first flatus within $48 \mathrm{hr}$ of operation. The analysis of cumulative frequencies revealed that there was no statistically significant difference between times of first flatus between two groups of patients (Figure 2).

Mean hospital stay for group 1 was 7.52 days (range: 3-14) and that for group 2 was 6.53 days (range 3-11). At any point of time, significantly smaller proportion of group 2 patients were in the hospital, compared with group 1 patients, as evidenced by the Kaplan-Meier curves (Spearman's correlation coefficient: -0.22; $\mathrm{P}<0.05$ ) (Figure 3).

NG tube had to be reinserted, according to predetermined indication in 3 patients in group 1 , and 4 patients in group 2; but the difference was not significant (P: 0.43) (Table 3 ). The symptoms, however, were alleviated in all cases after reinsertion. 


\begin{tabular}{|llll|}
\hline \multicolumn{4}{|l|}{ Table 3. Postoperative complications by study groups. } \\
\hline Complications & Group $\mathbf{~ n ~ ( \% )}$ & Group 2 $\mathbf{n}(\%)$ & Correlation coefficient (P value) \\
Gastric upset & $21(34.4 \%)$ & $21(38.8)$ & $-0.46(0.38)$ \\
Wound complication & $16(10.2)$ & $11(20.3)$ & $-0.06(0.30)$ \\
Respiratory complications & $12(19.6)$ & $11(20.3)$ & $-0.00(0.30)$ \\
Anastomotic leak & $2(4.5)$ & $2(5.1)$ & $-0.01(0.64)$ \\
NGT reinsertion & $3(4.9)$ & $4(7.4)$ & $(0.43)$ \\
\hline
\end{tabular}

\section{DISCUSSION}

To date in English literature, three meta-analyses have attempted to address the issue of post-operative NG decompression. The first such work by Cheatham et al included 10 clinical trials published till 1993, and concluded that routine use of NGI after elective surgery did not reduce the risk of ileus and aspiration, and rather prolonged recovery and hospital stay. ${ }^{10}$

Meta-analysis of 28 studies by Nelsen et al in 2005 included gastric cancer surgeries and emergency laparotomies. It clearly indicated that prophylactic use of NG decompression offers patients no benefit that would offset the discomfort and potential morbidity associated with its use. ${ }^{1}$ Observations by Vermeulen and associates were, too, similar to the earlier ones. ${ }^{11}$

Patients in both groups in the present study passed flatus within comparable times post-operatively. In fact, many studies on GI and non-Gl surgeries have found significantly earlier return of bowel function in non-intubated patients. Vermeulen's meta-analysis was based mostly on elective gastric and colorectal surgeries. ${ }^{11}$ Nelson et al drew their conclusions mostly from trials reporting on colorectal surgeries, where the mean time for first flatus ranged 3.1-4.9 days and 2.5-4.5 days for intubated and non-intubated patients respectively, with weighted mean difference of 0.46 days $(\mathrm{P}<0.001 ; 95 \% \mathrm{Cl}) .{ }^{1}$ Such a summary value could not be presented in the present study because of the categorical nature of the data collected; but lack of benefit from routine NGI was clearly evident.

The incidence of nausea, vomiting and abdominal distension have been variously reported, mostly in favor of NGI. In Nelson's analysis of 19 studies, majority of patients showed adverse effects with the routine use. ${ }^{1}$ In his comparison of gastric cancer surgery with versus without NGI, Lee observed more nausea in the no-NGI group with an RR of 2.77 at $95 \% \mathrm{Cl}^{12}$ Meta-analysis by Cheatham revealed a significantly greater abdominal distension ( $R R$ 1.34; $P=0.02)$ and vomiting ( $R R$ 1.45; $\mathrm{P}=0.005)$ in non-intubated patients. ${ }^{10}$ Reporting on colorectal surgeries, Wolff observed that frequency of nausea, vomiting and abdominal distension was significantly greater in patients whose NG tubes were removed in the recovery room. ${ }^{6}$ In the present study, however, continuation of NGI after surgery did not reduce the risk of gastric upset: an amalgam of nausea, vomiting and abdominal discomfort in this report (RR 0.88, OR 0.82; $\mathrm{P}=0.34$ ).

The most common problem with omission of NGT is gastric upset, necessitating subsequent insertion of a tube at times. Schwartz, Burg and others have reported that at most only $10 \%$ of no-NGI patients and $2-5 \%$ of routinely decompressed ones, required reinsertion subsequently. $6,10,13,14$ In the present study, $92.8 \%$ of patients not having routine postoperative NG decompression never required one. Five percent of patients undergoing routine postoperative nasogastric decompression required reinsertion of the tube. For every patient who required postoperative NGI, at least 14 patients could be spared one. Cheatham et al have comparably stated this number to be $20 .{ }^{10}$

Both the groups had similar rates of gastric upset and reinsertion of tubes, showing that routine NG decompression neither prevents the development of nausea, vomiting or abdominal discomfort and distension; nor precludes the need for tube replacement once it is discontinued.

In Nelson's review, the risk of respiratory complications was comparably different between the two groups in subgroup analyses of upper $\mathrm{Gl}$ and colorectal surgeries. However, the overall benefit approached statistical significance (RR 1.35; $\mathrm{P}=0.07) .{ }^{1}$ In the present study, $\mathrm{NGI}$ did not seem to reduce the risk of respiratory complications $(P=0.30)$.

In fifteen studies analyzed by Nelson, the difference in rates of wound infection was not significant, a finding similar to present study where NGI after operation did not reduce the rate of wound infection. ${ }^{1}$ Similar results were also obtained by Cheatham et al. ${ }^{10}$

Many researchers have reported a consistent reduction in the length of hospital stay with omission of NGT. ${ }^{10,17,18}$ Although Friedman et $\mathrm{al}^{15}$ reported that the length of hospital stay was increased (weighted mean difference: 
-0.90 days; $95 \% \mathrm{Cl}$ ) in no-NG group in patients who underwent surgery for abdominal aortic aneurysms, the generalization of the result to other GI surgeries needs careful evaluation. In the present study, too, length of hospitalization showed statistical difference in favor of no-NGI, with $\mathrm{P}<0.05$.

One hundred and two patients with a newly constructed anastomosis were reviewed by Cunningham et al. who showed that the omission of NGT did not result in any higher incidence of anastomotic complications $10 \%$ with NGT and $2 \%$ without $[\mathrm{P}>0.25]) .{ }^{16}$ Nelson reported that there was no significant difference in leak between the two groups ( $P=0.7$; OR 0.86$)$, quite comparable to the present study $(\mathrm{P}=0.64$; OR 0.89).

Nevertheless, postoperative NGI still seems to be a common practice despite a clear lack of support of evidence. Not employing a routine nasogastric tube does not mean never using the tube. The emphasis is on avoiding routine, empirical use. Some patients with intractable gastric upset were evidently benefited with the use of NGI. The call is for a judicious use of NGI, as opposed to what is being practiced: the routine, reflex use in all patients after emergency laparotomy.
However there were certain limitations of this study. Because of the typical nature of the intervention, blinding of either patients or observers would be impossible, and was never attempted. The obvious introduction of surgical judgment into the decision to exclude patients with dense fibrotic adhesions might have been a source of selection bias. In an attempt to offset the reporting bias due to subjectivity and an inherent imprecision, time of first flatus was pooled into various time ranges. No attempt was made to grade the other primary endpoint, the gastric upset. Some parameters, like duration of peritonitis or obstruction that would have contributed to return of bowel function, were not considered.

\section{CONCLUSIONS}

This study has shown that the prophylactic nasogastric decompression following emergency laparotomy is ineffective in achieving any of the intended goals of hastening the return of bowel function, relieving gastrointestinal discomfort, reducing various postoperative complications and reducing hospital stay. Routine use of NGT after all emergency laparotomy must thus be questioned.

\section{REFERENCES}

1. Nelson R, Tse B, Edwards S. Systematic review of prophylactic nasogastric decompression after abdominal operations. Br J Surg. 2005;92:673-80.

2. Sands DR, Wexner SD. Nasogastric tubes and dietary advancement after laparoscopic and open colorectal surgery. Nutrition. 1999;15:347-50.

3. Witting MD. 'You wanna do what?' modern indications for nasogastric intubation. J Emerg Med. 2007;33:61-4.

4. Gerber A. An appraisal of paralytic ileus and the necessity of postoperative gastrointestinal suction. Surg Gynecol Obstet. 1963;117:294-6.

5. Bauer JJ, Gelernt IM, Salky BA et al. Is routine postoperative nasogastric decompression really necessary? Ann Surg. 1985;201(2):233-6.

6. Wolff BG, Pemberton JH, van Heerden JA et al. Elective colon and rectal surgery without nasogastric decompression. Ann Surg. 1989;209:670-3.

7. Mattei P, Rombeau JL. Review of pathophysiology and management of postoperative ileus. World J Surg. 2006;30:1382-91.

8. Kehlet H, Williamson R, Buchler MW et al. A survey of perceptions and attitudes among European surgeons towards the clinical impact and management of postoperative ileus. Colorectal Dis. 2005;7(3):245-50.

9. Kehlet H, Buchler MW, Beart RW Jr et al. Care after colonic operation- is it evidence-based? Results from a multinational survey in Europe and the United States. J Am Coll Surg. 2006;202(1):45-54
10. Cheatham ML, Chapman WC, Key SP et al. A Meta-analysis of selective versus routine nasogastric decompression after elective laparotomy. Ann Surg. 1995;221:469-78.

11. Vermeulen $\mathrm{H}$, Storm-Versloot $\mathrm{MN}$, Busch ORC et al. Nasogastric intubation after abdominal surgery. A meta-analysis of recent literature. Arch Surg. 2006;141:307-14.

12. Lee JH, Hyung WH, Noh SH. Comparision of gastric cancer surgery with versus without nasogastric decompression. Yonsei Med J. 2002;43:451-6.

13. Schwartz CI, Heyman AS, Rao AC. Prophylactic nasogastric tube decompression: Is its use justified? South Med J. 1995;88: 825-30.

14. Burg R, Geigle CF, Faso JM et al. Omission of routine gastric decompression. Dis Col Rect. 1978;21:98-100.

15. Freidman SG, Sowerby SA, Pin CAD et al. A prospective randomized study of abdominal aortic surgery without postoperative nasogastric decompression. Cardiovasc Surg. 1996;4:492-4.

16. Cunningham J, Temple WJ, Langevin JM et al. A prospective randomized trial of routine postoperative nasogastric decompression in patients with bowel anastomosis. Can J Surg. 1992;35:629-31.

17. Montgomerry RC, Bar-Natan MF, Thomas SE et al. Postoperative nasogastric decompression: a prospective randomized trial. South Med J. 1996;89:1063-6.

18. Pearl ML, Valea FA, Fischer M et al. A randomized controlled trial of postoperative nasogastric tube decompression in gynecologic oncology patients undergoing intra-abdominal surgery. Obstet Gynecol. 1996;88:399-402. 